\title{
Mechanical Phenotyping of Mouse Embryonic Stem Cells: Increase in Stiffness with Differentiation
}

\author{
Anand Pillarisetti, ${ }^{1, \star}$ Jaydev P. Desai, ${ }^{1}$ Hamid Ladjal, ${ }^{2}$ Andrew Schiffmacher, ${ }^{3}$ \\ Antoine Ferreira, ${ }^{2}$ and Carol L. Keefer ${ }^{3}$
}

\begin{abstract}
Atomic force microscopy (AFM) has emerged as a promising tool to characterize the mechanical properties of biological materials and cells. In our studies, undifferentiated and early differentiating mouse embryonic stem cells (mESCs) were assessed individually using an AFM system to determine if we could detect changes in their mechanical properties by surface probing. Probes with pyramidal and spherical tips were assessed, as were different analytical models for evaluating the data. The combination of AFM probing with a spherical tip and analysis using the Hertz model provided the best fit to the experimental data obtained and thus provided the best approximation of the elastic modulus. Our results showed that after only 6 days of differentiation, individual cell stiffness increased significantly with early differentiating mESCs having an elastic modulus two- to threefold higher than undifferentiated mESCs, regardless of cell line (R1 or D3 mESCs) or treatment. Singletouch (indentation) probing of individual cells is minimally invasive compared to other techniques. Therefore, this method of mechanical phenotyping should prove to be a valuable tool in the development of improved methods of identification and targeted cellular differentiation of embryonic, adult, and induced-pluripotent stem cells for therapeutic and diagnostic purposes.
\end{abstract}

\section{Introduction}

$\mathbf{E}$ MBRYONIC STEM CELLS (ESCs) have the unique capability to differentiate into a diverse array of specialized cell types. Consequently, human embryonic stem cells have strong potential for therapeutic use in treatment of disease (e.g., heart disease, Parkinson's disease, and spinal cord injuries). Conventionally, differentiating precursor and differentiated cells are distinguished from undifferentiated stem cells by (1) antibody staining of specific protein markers, (2) morphometric parameters (cell shape/structure), and (3) transgenic reporter markers. Cell sorting based on antibody staining involves labeling specific cell lineages with fluorescent antibodies and then purifying them by fluorescenceactivated cell sorting (FACS) (Bourne et al., 2004). This method is time-consuming, expensive, and damaging to the cells. Some terminally differentiated cells can be identified by morphometric parameters, but these parameters cannot identify precursor cells (Odorico et al., 2001). Transgenic re- porter markers utilize cell-specific promoters, which control the expression of reporter proteins for defined cell states. This method requires genetic modification of cells (Christoforou et al., 2008; Donovan and Gearhart, 2001; Kolossov et al., 1998). Moreover, there is limited availability of reliable markers for early lineage precursors (Nagano et al., 2008; Smith, 2001), and gene silencing can be an issue (Stewart et al., 2008). Thus, generation of pure populations of defined cell types remains a challenge for stem cell biologists. For clinical applications, noninvasive methods of efficient cell characterization are needed to identify the desired cell type. Conversely, the ability to identify early dedifferentiating cells during induced pluripotency treatments for patient specific therapy would also be beneficial. We propose that the mechanical properties of stem cells could be used for such purposes.

Mechanical properties of cells, such as elasticity and viscoelasticity, directly reflect cellular composition, internal structure (cytoskeleton, organelles, etc.), and external

\footnotetext{
${ }^{1}$ Robotics, Automation, Medical Systems (RAMS) Laboratory, University of Maryland, College Park, Maryland.

${ }^{2}$ Institut Prisme, ENSI de Bourges, France.

${ }^{3}$ Department of Animal and Avian Sciences, University of Maryland, College Park, Maryland.

${ }^{*}$ Current address: Sensata Technologies, Inc., Attleboro, MA 02703.
} 
interactions (cell-cell and/or cell-surface). Alterations in the mechanical properties of cells, therefore, can be used to detect changes in their cellular composition. Danti et al. (2007) used force modulation microscopy (FMM) to detect variations in mechanical properties of human mesenchymal stem cells (hMSCs). However, FMM imaging technique is not quantitative, and it can detect only relative and qualitative elastic modulus differences between different cell surfaces. Several researchers (Docheva et al., 2008; Titushkin and Cho, 2007; Yourek et al., 2007) have also studied the mechanical properties of hMSC using Atomic Force Microscopy (AFM). Their results demonstrated that the cytoskeleton plays an important role in the differentiation of hMSC. Similarly, changes in the cytoskeletal (F-actin) and nuclear matrix (lamin) composition have been observed to affect both cytoskeletal and nuclear stiffness in ESCs (Chowdhury et al., 2010; Pajerowski et al., 2007). Each of these studies looked at only one component, that is, either the nuclear stiffness using rheological aspiration (nuclear deformation) or cytoskeletal stiffness using the twisted bead approach (cytoskeletal resistance). In the cytoskeletal resistance studies an increase in F-actin density in mouse ESCs was correlated with an increase in cell stiffness (Chowdhury et al., 2010), whereas in the rheological studies (Pajerowski et al., 2007), in which nuclear deformation (plasticity) was assessed in human ESCs, adult hMSCs, and epithelial cells, the deformability of nuclei was affected by lamin composition of the nuclear matrix. Stem cells that did not express lamin A/C possessed more fluid nuclei than differentiated cells that contained lamin A/C.

Because ESCs are characterized by their large nuclear: cytoplasmic ratio, such that the bulk of the cell consists of the nucleus with a small rim of cytoplasm, we hypothesized that this change in nuclear physical plasticity could be detected by directly probing the cell using a single touch (indentation) approach. This approach would require less cell manipulation than the rheological or twisted-bead approach and would be more amenable to scale-up efforts. Furthermore, single-touch probing is less likely to induce unwanted cell responses to repeated mechanical stimuli (Chowdhury et al., 2010). Mouse ESCs (mESCs) provided a well-characterized model for use in our studies. The mESC can be grown on a gelatin matrix in the absence of feeder cells, thus eliminating any confounding effects from the feeder cells. The cytokine LIF is provided to maintain pluripotency: removal of LIF results in spontaneously differentiation toward a neural lineage (Chambers and Smith, 2004). During differentiation endogenous markers of pluripotency, such as the transcription factors Nanog and Oct4 (POU5F1), will disappear over time and the morphological characteristics of the cells will change. Usually this differentiation process is followed over a time period of 7 to 15 days and longer in order to identify precursor cells in the lineage of interest. We conducted studies on undifferentiated and early differentiating mESCs within a 6-day time frame using an AFM system. AFM tip geometry and various analytical models were evaluated to determine the appropriate model to characterize the local cell stiffness. In these studies, we were able to detect changes in local cell stiffness in mESCs probed during these early initial stages of differentiation and prior to gross morphological changes in cell shape or size.

\section{Materials and Methods}

\section{Experimental setup}

The Atomic Force Microscope (MFP-3D-BIO ${ }^{\mathrm{TM}}$, Asylum Research, Santa Barbara, CA) system consisted of a scan head integrated with a phase contrast module and an inverted microscope (Model: TE2000U, Nikon, Inc., Melville, NY). The microscope was mounted on an active vibration isolation table (Herzan, Laguna Hills, CA). The phase contrast module enabled visualization of low-contrast transparent cells in the fluid medium. The XY stage allowed the user to position the cell beneath the AFM cantilever tip (Fig. 1 ). The entire setup was enclosed in an acoustic isolation chamber to prevent ground vibrations and acoustic noise from interfering with the AFM measurements. The $x$ - and $y$ axes range of the scan head was $90 \mu \mathrm{m}$. The $z$-axis scan range was customized at $40 \mu \mathrm{m}$. The scan head had a piezoelectric scanner, which moved the cantilever in the $z$-direction toward the cell. In addition, the head had a photodiode that detected the deflection of the cantilever when the tip contacted and deformed the cell. This AFM system, which has the capability to measure forces in the range of $\mathrm{pN}-\mathrm{nN}$, was used to obtain force and cell deformation data from individual cells by single-touch (indentation), single-cell probing, rather than running scans across multiple cells in a monolayer. The cantilever was moved by the piezoelectric scanner in $z$ direction toward the cell. The deflection of the cantilever was detected by a photodiode when the tip came in contact with the cell. When the tip of the cantilever was in contact with the cell, the initial cantilever deflection, $\left(d_{0}\right)$, and initial cantilever movement in $z$ direction $\left(z_{0}\right)$ were stored through the IGOR software interface (Asylum Research, Inc.). As the cantilever moved further in the $z$ direction and deformed the cell, the final cantilever deflection $\left(d_{1}\right)$, and the cantilever movement $\left(z_{1}\right)$, were obtained (Pillarisetti et al., 2008). Prior to any experiments, cantilevers were calibrated to determine resonant frequency and spring constant (see Ladjal et al., 2010), since for each particular tip geometry, the stiffness of the cantilever plays a crucial role in determining the elastic modulus of the cell. After conducting several trials, a silicon nitride cantilever with spring constant of $0.06 \mathrm{~N} / \mathrm{m}$ (Novascan Technologies, Inc., Ames, IA) was chosen for indenting live ESCs, whereas a relatively stiffer silicon cantilever $(k \sim 2 \mathrm{~N} /$ $\mathrm{m}$, Novascan Technologies, Inc.) was chosen for indenting fixed ESCs.

The cell indentation $(\delta)$ and the force $(F)$ needed to result in the set indentation distance were used to characterize the mechanical property of the cell. By modeling the system (AFM cantilever and the cell) as two linear springs in series, the force experienced by the cell could be obtained from the product of spring constant of the cell and the cell indentation:

$$
F=k d
$$

where $k$ is the spring constant and $d$ is cantilever deflection. Specifically, at the beginning of each experiment, the probe tip was touched to the dish surface near a cell and the spring constant determined through the software (Ladjal et al., 2010). The spring constant was computed by the thermal noise method (Hutter and Bechhoefer, 1993), which takes into consideration the sensitivity factor of the cantilever. As the 


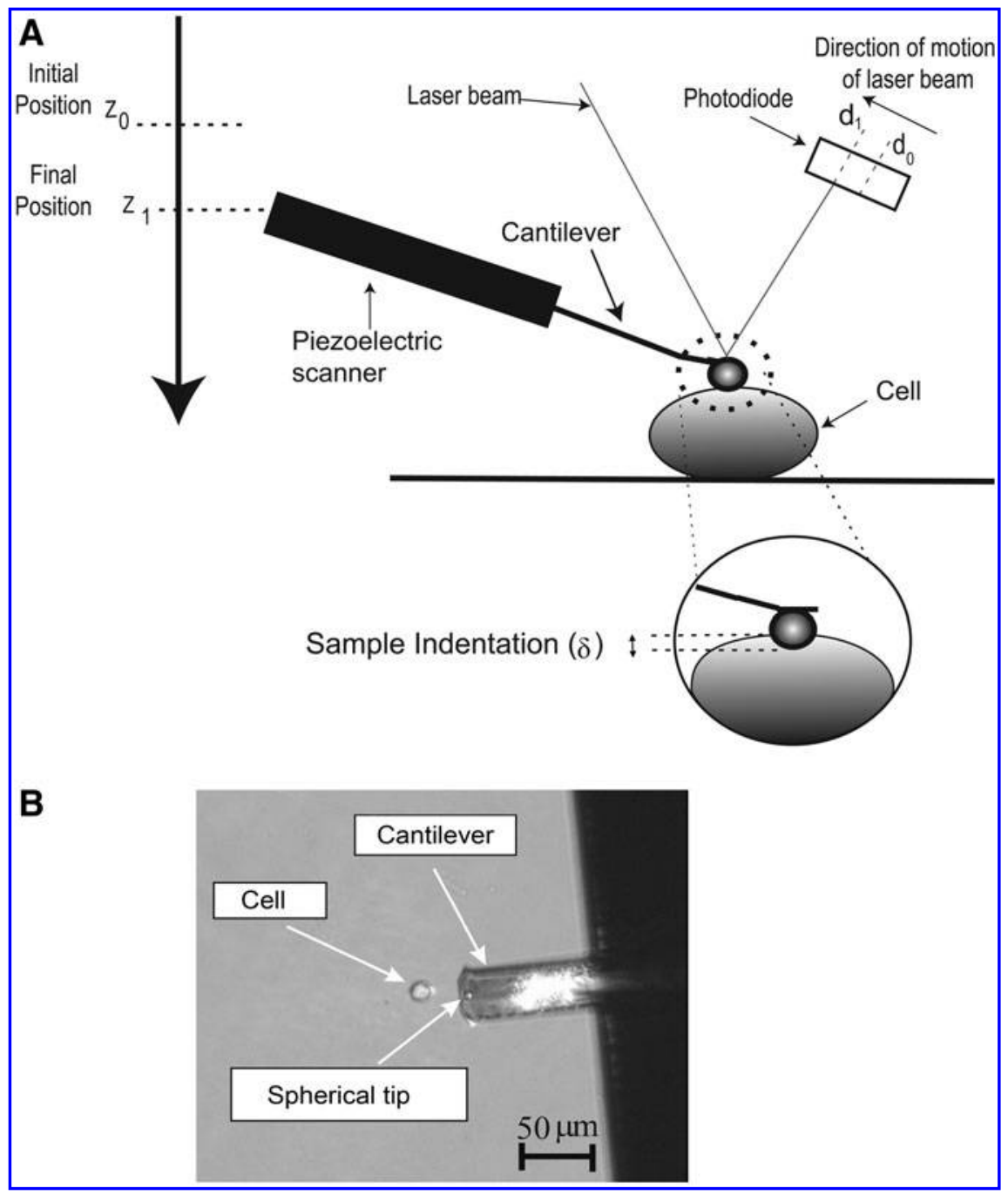

FIG. 1. (A) Schematic of AFM cantilever interacting with a cell. Point of contact: $d_{0}$ and $z_{0}$ represent the initial cantilever deflection and cantilever movement, respectively. Cell indentation: the cantilever moves further from the point of contact and indents the cell: $d_{1}$ and $z_{1}$ represent the final cantilever deflection and movement, respectively. (B) Cantilever with a spherical probe poised near an individual mESC.

force experienced by the cell was equal to the force exerted on the cantilever, $F$ was also obtained through the IGOR software interface.

Force-indentation data can be obtained by applying a certain known force on the cell and recording the subsequent cell indentation value using the AFM system. However, this method involves an extra step of determining the force required to indent a particular cell state (live/fixed and undifferentiated/differentiating) before the experiments and thus is time-consuming. Furthermore, the same force value may indent cells in different physiological states by either a negligible amount or result in damage to the cell. Hence, the standard experimental procedure that we used was to indent all the cells by the same distance value and record the corresponding indentation forces. However, the indentation range depends on the cell height, which was calculated from force-displacement curves obtained on a hard surface near the cell and on the cell surface. The mESC heights were approximately in the range of $7-17 \mu \mathrm{m}$ (Ladjal et al., 2010). The cell indentation range was chosen to be $2-2.5 \mu \mathrm{m}$. As the indentation range becomes a significant fraction of the cell height it is necessary to develop an appropriate contact model that can account for the observed force-indentation data (Ladjal et al., 2010). The cell was deformed by using the "indentation trigger" (custom made by Asylum Research) based on the equation

$$
\delta=\left(z_{1}-z_{0}\right)-\left(d_{1}-d_{0}\right)
$$

in which the parameters $d_{0}$ and $z_{0}$ represented the initial cantilever deflection and cantilever movement ( $z$ direction), respectively, when the AFM tip contacted the cell (Fig. 1A). Similarly, $d_{1}$ and $z_{1}$ represented the final cantilever deflection and movement, respectively, when the AFM tip deformed the cell. Indentation trigger is a constant value by which the AFM cantilever deforms the cell and then retracts from it. Substituting the actual cantilever deflection into the force formula, we get:

$$
F=k\left(d_{1}-d_{0}\right)
$$

The difference between the actual cantilever movement, $\mathrm{z}$, and actual cantilever deflection, $d$, represents the cell indentation, $\delta$. The cell indentation $(\delta)$ and the force $(F)$ can be used to determine the mechanical behavior of the cell using an appropriate analytical model. 


\section{Analytical models}

Numerous researchers have used the Hertz-Sneddon model to characterize the mechanical properties of cells using AFM (Collinsworth et al., 2002; Touhami et al., 2003; Vinckier and Semenza, 1998; Weisenhorn et al., 1993). The Hertz theory can be used to study the contact mechanics between a spherical tip and a cell (Johnson, 1982), although the Bilodeau model (Bilodeau, 1992) can be used to compute the mechanical properties of cells when using pyramidal AFM tips (Alcaraz et al., 2003; Rosenbluth et al., 2006). Other models take into consideration other shaped probes and issues with adhesion forces (Maugis, 2000; Sneddon, 1948). We have selected the Bilodeau and Hertz models for studies characterizing the relationship between alterations in cell stiffness and cell differentiation status (for more details on model analysis, see Ladjal et al., 2010; Pillarisetti, 2009). Furthermore, we assessed the force (loading and unloading) versus time profiles for each $\mathrm{mESC}$ indentation to determine whether adhesion force existed between the tip and the sample.

\section{Cell preparation}

Mouse ESC lines, R1 and D3 (ATCC No. SCRC-1011 and CRL-1934, American Type Culture Collection, Manassas, VA), were grown on $0.1 \%$ gelatin-coated plates in the absence of feeder cells in DMEM supplemented with 15\% FBS and $1000 \mathrm{IU} \mathrm{LIF} / \mathrm{mL}$ (+LIF) as previously described (He et al., 2008). Differentiation was induced by one of the following methods: (1) removal of LIF from the medium (-LIF) (Sharova et al., 2007) or (2) removal of LIF from the medium plus the addition of $0.1 \mathrm{mM}$ ascorbic acid (-LIF+AA), which induces differentiation toward a cardiac lineage (Takahashi et al., 2003). Prior to AFM experiments, cells were dispersed using trypsin and plated on $60-\mathrm{mm}$ gelatin-coated tissue culture dishes. Cells were cultured in the appropriate medium (+LIF, - LIF or - LIF+AA) for $5 \mathrm{~h}$ to allow for attachment, after which live cells were either assessed immediately using AFM or fixed with $4 \%$ formaldehyde for later assessment by AFM or immunocytochemistry. During AFM assessment, the culture medium for live cells was replaced with a medium buffered for room atmospheric conditions and supplemented with bovine serum albumin (Emcare, ICPbio, Ltd., Auckland, New Zealand). For fixed preparations, ESCs were treated with $4 \%$ formaldehyde for $10 \mathrm{~min}$, washed, and stored in Dulbecco's phosphate-buffered saline (DPBS) until subsequent assessment by AFM within $72 \mathrm{~h}$ of fixation. The fixation process kills the cell but also preserves the cell structure at that point in time and development. For consistency, fixed cells were also assessed in protein supplemented Emcare. All cells were labeled with the DNA stain Hoechst 33342 in order to identify the cell nucleus during AFM assessment. We performed AFM experiments on live as well as fixed mESCs to validate the correlation between them. This was done primarily because when logistics prove difficult to maintain live cells, the experiments could be performed on fixed cells, but only if the results obtained with live cells were shown to parallel that of fixed cells.

The expression of the pluripotency-related transcription factor, Nanog, and nuclear matrix lamins were assessed in fixed undifferentiated and differentiating cells by immunocytochemistry using protocols described previously (He et al.,
2006). Fixed and permeabilized ESCs were incubated with rabbit antimouse NANOG antibody (cat. no. AB5731, Chemicon, Temecula, CA) and visualized using donkey antirabbit secondary antibody conjugated with FITC (cat. no. AP182F, Chemicon). Nuclear lamin A/C proteins were labeled using mouse antinuclear lamin A/C (cat. no. 39288, Active Motif, Carlsbad, CA) visualized using Chromeo ${ }^{\mathrm{TM}} 546$ Goat antimouse IgG (cat. no. 15033 Active Motif). F-actin was labeled with phalloidin-350 (Invitrogen, Carlsbad, CA) according manufacturer's instructions. DNA was stained with DAPI (Actif Motif).

\section{Cell probing}

In each set of the experiments, single indentation studies were conducted on at least 10 control cells (undifferentiated) and 10 treated cells (differentiating). In experiments in which indentation studies were conducted on both live and fixed cells (R1-LIF removal), all four conditions (live: fixed: undifferentiated: early differentiating) were assessed for the same cell preparation. Cells were dispersed and allowed to attach as single cells on the gel-coated plates so that each cell could be assessed individually. Previously, large variations in elastic modulus of mESCs were observed, which could be due to variation in the stage of the cell cycle at the time of measurements (Pillarisetti et al., 2008 ). Hence, in the present work we focused on mESCs in the interphase stage of the cell cycle process. Cells in interphase were identified using the nuclear dye, Hoechst 33342. The interphase nucleus takes up the main volume of the mESCs; there is only a thin rim of cytoplasm surrounding the nucleus.

To calibrate the spring constant of the cantilever probe, a single force curve was taken at the beginning of each experiment by touching the probe tip onto the tissue culture plate surface next to a cell. The probe tip was then aligned over the cell nucleus and the indentation force-curve profile recorded. The approach velocity was $1.98 \mu \mathrm{m} / \mathrm{sec}$ and the force curve resolution was 2000 data points/sec. After preliminary studies to determine the preferred tip geometry, additional independent cell culture replicates were performed for each treatment and cell line using a probe with a spherical tip. In all the experiments, the cell indentation range was 2-2.5 $\mu \mathrm{m}$ (Ladjal et al., 2010; Pillarisetti, 2009).

\section{Statistics}

In the studies comparing the Bilodeau and the Hertz models, the least-square method was used to fit the analytical models to the force versus indentation profiles of mESCs $\left(R^{2}\right.$ value was calculated as the square of the correlation coefficient). Statistical analysis of the elastic modulus values was performed using the Kruskal-Wallis and the Mann-Whitney tests. The level of significance ( $\alpha$-value) was chosen to be 0.05 ; hence, results were considered significant when $p<0.05$.

\section{Results \\ Cell culture}

Undifferentiated mESCs display the following characteristics: high nuclear/cytoplasmic ratio, formation of compact colonies with smooth edges, and the expression of pluripotency-related transcription factors, such as Nanog. Removal of LIF and/or addition of differentiating inducing 
conditions will cause the ESCs to differentiate into a variety of cell types. However, this differentiation process occurs over an extended time period: specific precursor cells can be identified generally after 7-21 days. In our studies, early differentiating cells ( 6 days after removal of LIF) showed few morphological changes compared to undifferentiated, although cell colonies were somewhat less compact (Fig. 2A and C). Immunostaining of Nanog protein demonstrated that the transcription factor was still present in the nuclei of early differentiating ESCs (Fig. 2D). Transgenic R1 ESCs, which expressed a nuclear-localized green fluorescent protein (nlGFP) driven by the bovine Nanog promoter (bNanognlGFP), were assessed for expression of the nuclear matrix proteins, lamin $\mathrm{B}$, and lamin $\mathrm{A} / \mathrm{C}$, using immunocytochemisty. The percentage of cells expressing the nuclearlocalized GFP reporter in the bNanog-GFP transgenic cells did appear to decrease in differentiating cells compared to undifferentiated $[11 \%(25 / 237)$ vs. $49 \%(156 / 317)$, respectively, $p<0.0001$, Fisher's Exact Test]. The percentage of undifferentiated cells expressing the bNanog-nlGFP was lower than expected in the undifferentiated population (49\%) due to gene silencing of the transgene, which can be an issue with reporter genes (Stewart et al, 2008). The percentage of cells that stained immunocytochemically for endogenous lamin A/C protein increased from $14 \%(32 / 230)$ in undifferentiated cells to $71 \%(103 / 144)$ in differentiating cells $(p<0.0001$, Fisher's Exact Test) (Fig. 2F and H). All cells expressed lamin B. The pattern of F-actin staining changed slightly with early differentiating transgenic R1 cells (bNanog-nlGFP) having more defined stress fibers, as shown in Fig. 2I-L).

\section{Analytical modeling}

The Bilodeau and Hertz models were evaluated based on the geometry of the tip used in each set of cell indentation studies: (1) Bilodeau model for ESCs indented by a pyramidal tip, and (2) Hertz model, as well as the capsule model, for ESCs indented by a spherical tip. These mathematical models are used to estimate cell stiffness. The capsule model considers the contribution of stretching and bending of the cell membrane to cell stiffness. Furthermore, we determined whether adhesion force existed between the tip and the sample in order to decide whether or not to consider the Johnson-Kendall-Roberts (JKR) and Derjagin-MullerToropov (DMT) models of solids adhesion (Pillarisetti, 2009). Both live and formaldehyde-fixed cells were assessed.

Medium used during probing was supplemented with protein to decrease any adhesion between probe tip and cell. Adhesion forces were observed for only a few samples in force (loading and unloading) versus time profiles in any of the studies (data not shown; for an example profile, see Ladjal et al., 2010). The adhesion detected in these samples could be due to the wear of the spherical probe (Kwon et al., 2007). In those few samples, this force was negligible (less than 0.05 times the maximum indentation force) compared to the adhesion force (more than 0.1 times the maximum indentation force) observed by other researchers (Cao et al., 2005; Girot et al., 2006; Kwon et al., 2007). Hence, the adhesion force was not considered in the subsequent analyses, and the JKR and DMT models were not applicable (Ladjal et al., 2010; Pillarisetti, 2009).

\section{Pyramidal tip}

The force-indentation profiles were obtained from live undifferentiated and early differentiating mESCs probed by a silicon nitride cantilever (PNP-DB, quadrilateral tip). The Bilodeau model was used to fit the profiles. The $R^{2}$ value for the fit was 0.90 and 0.85 for live undifferentiated and differentiating mESCs, respectively. The force-indentation profiles were also obtained from fixed undifferentiated and early differentiating mESCs probed by a silicon cantilever (AC 240TS, tetrahedral tip). Similar to live mESCs, the Bilodeau model was used to fit the profiles. The $R^{2}$ value for the fit was 0.83 and 0.80 for fixed undifferentiated and differentiating mESCs.

\section{Spherical tip}

Figure $3 \mathrm{~A}-\mathrm{B}$ shows the force-indentation profiles obtained from live undifferentiated and early differentiating mESC s probed by a spherical tip. The $R^{2}$ value obtained with Hertz model was 0.95 and 0.87 for live undifferentiated and differentiating mESCs, respectively. On the other hand, low $R^{2}$ values were obtained with capsule model with 0.64 and 0.55 for live undifferentiated and differentiating mESCs, respectively (data not shown). Figure 3C-D shows the forceindentation profiles obtained from fixed undifferentiated and early differentiating mESCs probed by a spherical tip. The $R^{2}$ value obtained with Hertz model was 0.90 and 0.91 for fixed undifferentiated and differentiating mESCs, respectively. The $R^{2}$ values obtained with capsule model were 0.42 and 0.55 for fixed undifferentiated and differentiating mESCs, respectively (data not shown).

\section{Mechanical properties-elastic modulus}

As described above, the Bilodeau model appropriately described the mechanical behavior of mESCs probed with a pyramidal tip, whereas the Hertz model appropriately described behavior of cells probed with a spherical tip. The capsule model, which considers the stretching and the bending of the cell membrane, was rejected. One explanation for this rejection could be that the mESC mechanics as determined by AFM for live and fixed cells are influenced more by the compression of the cell interior (e.g., cytoskeleton/ nucleus) than that of the cell membrane. Therefore, the Bilodeau and Hertz models were used to compute the elastic modulus (stiffness) of each mESC (live and fixed) sample probed by pyramidal and spherical tip, respectively. In these experiments, differentiation of mESCs (R1 cell line) was induced by LIF removal. For live mESCs measured using a pyramidal tip, the average elastic modulus (EM) of undifferentiated cells was significantly lower than differentiating cells, $1.49 \pm 0.09$ kilopascal $(\mathrm{kPa})$ versus $16.069 \pm 1.48 \mathrm{kPa}$, respectively ( $\mathrm{kPa}$ presented as mean $\pm \mathrm{SE}$ ), with a $p$-value of 0.0002. For fixed mESCs, the average EM of undifferentiated cells was also lower than differentiating cells, $35.185 \pm 3.04 \mathrm{kPa}$ versus $120.87 \pm 11.63$, respectively $(p$ value $=0.0002)$. Using a spherical tip, the average EM obtained for undifferentiated and differentiating live mESCs were significantly different $(p$-value $=0.0003), 0.2176 \pm 0.015$ and $0.4473 \pm 0.036 \mathrm{kPa}$, respectively. For fixed mESCs probed with a spherical tip, the average EM obtained for undifferentiated and differentiating was significantly different 


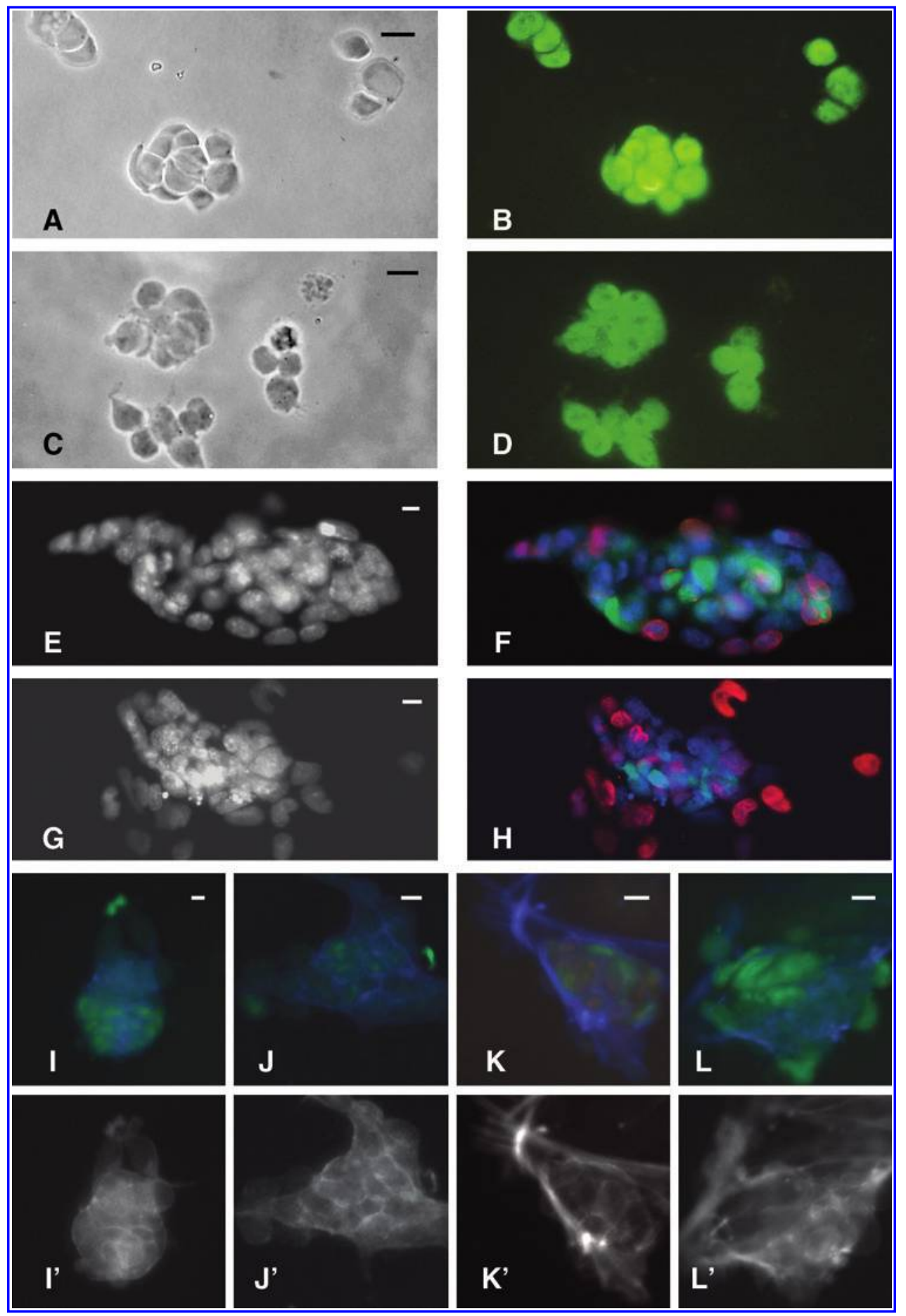

FIG. 2. Undifferentiated and differentiating mESCs. (A) Undifferentiated cells (brightfield). (B) Same cells as in A labeled with anti-Nanog primary antibody and FITC-conjugated secondary antibody. (C) Differentiating mESCs 6 days after removal of LIF and incubation in $0.1 \mathrm{mM}$ ascorbic acid (brightfield). (D) Same cells as in C labeled with anti-Nanog antibody and FITC conjugated secondary antibody. (E) Nuclei of undifferentiated mESCs stained with DAPI. (F) Same cells as in E stained with DAPI (blue) expressing the nuclear localized reported transgene (green, Nanog promoter-nlsGFP) and labeled with antilamin A/C primary antibody and Active-Motif Chromeo ${ }^{\mathrm{TM}} 546$ conjugated secondary antibody (red). (G) Nuclei of differentiating ESCs stained with DAPI. (H) Same cells as in G expressing the nuclear localized reporter transgene (green, Nanog promoternlsGFP) and labeled with antilamin A/C primary antibody and Active-Motif Chromeo ${ }^{\text {TM }} 546$ conjugated secondary antibody (red). (I, J) Undifferentiated mESCs expressing the nuclear localized reporter transgene (green; Nanog promoter-nlsGFP) with F-actin labeled with phalloidin-350 (blue). (K, L). Differentiating mESCs expressing the nuclear localized reporter transgene (Nanog promoter-nlsGFP) with F-actin labeled with phalloidin-350 (blue). K also labeled with anti-lamin A/C (red). (I'-L') Factin patterns for I-L. Bars $\approx 10 \mu \mathrm{m}$. 

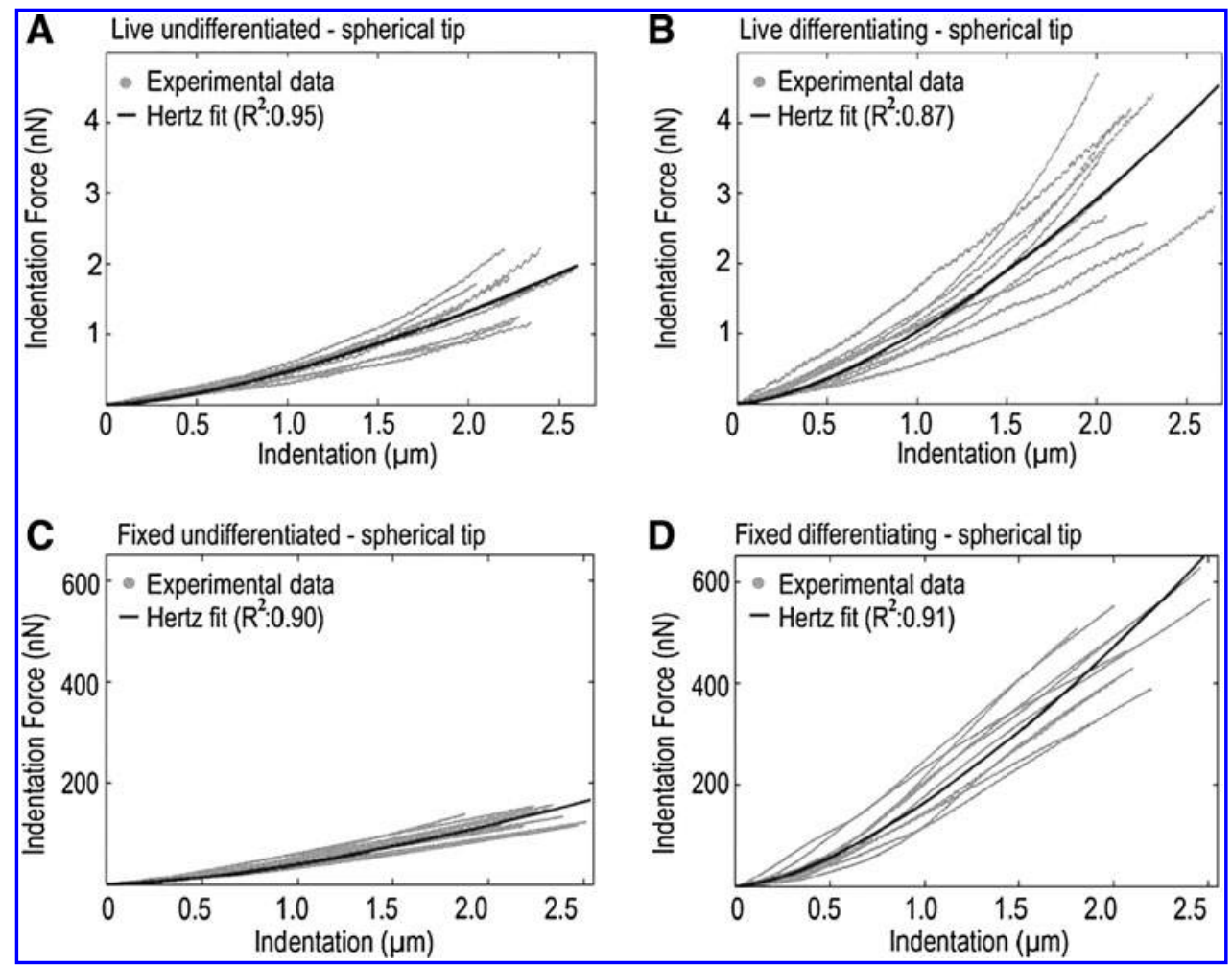

FIG. 3. Force $(\mathrm{nN})$ versus cell indentation $(\mu \mathrm{m})$ for undifferentiated and differentiating ESCs, live and fixed as indicated in each panel. Each grey line represents data obtained from an individual cell $(n=10$, each panel). Black lines are the expected results determined by the Hertz model for data obtained with a spherical tip. $\mathrm{R}^{2}$ equals the square of the correlation coefficient (adapted from Pillarisetti et al., 2009).

$(p$-value $=0.0002), 17.86 \pm 1.06$ and $73.51 \pm 4.28 \mathrm{kPa}$, respectively. Thus, for live and fixed cells, undifferentiated mESCs were suppler than differentiating mESCs, regardless of the tip geometry used for probing. The mean elastic modulus obtained with a spherical tip was less than the mean elastic modulus obtained with a pyramidal tip for undifferentiated and differentiating mESCs in both live and fixed cells. This decrease in mean value could be due to the lower stress concentration as compared to the high stress concentration in the cell induced by probing with a sharper tip (Rico et al., 2005). The larger area of spherical tip-to-cell contact results in an averaging of the local modulus variation compared to that measured with a pyramidal tip. Thus, we hypothesize that the elastic modulus obtained with a spherical tip better predicts the global mechanical property of mESCs and additional studies were performed using a spherical tip (Table 1).

Differentiation was induced by LIF removal in both R1 and D3 cell lines and by ascorbic acid treatment in R1 cells to demonstrate that the change in elastic modulus was not specific to one cell line or treatment. The average values for the elastic modulus obtained using a spherical tip are shown in Table 1. The results obtained with live R1 cells parallel that of fixed R1 cells, that is, undifferentiated mESCs are suppler compared to differentiating mESCs for both live and fixed cells. In initial studies fixed cells were assessed within $72 \mathrm{~h}$ of fixation; however, as time between fixation and analysis increased, the stiffness of the cells increased. Therefore, AFM probing was performed within $24 \mathrm{~h}$ of fixation in all studies except for the initial R1-LIF removal study. Crosslinking of proteins by formaldehyde fixation increased the cell stiffness by 14 - to 19 -fold for cells measured with in $24 \mathrm{~h}$ of fixation (Table 1). Nevertheless, in all studies early differentiating cells were significantly less supple compared to controls (undifferentiated mESCs), irrespective of cell line (R1 or D3) or induction method (LIF removal or ascorbic acid treatment).

\section{Discussion}

In this study, we performed indentation studies with an AFM on undifferentiated and early differentiating mESCs (live and fixed cells) and used the information obtained to select an appropriate analytical model to characterize the mechanical behavior of an individual mouse embryonic stem cell. Based on the experimental data, we determined that the Bilodeau and Hertz model appropriately describe the mechanical behavior of mESCs probed by a pyramidal and a spherical tip, respectively. We confirmed our hypothesis that the mechanical property of undifferentiated mESCs, as defined by the elastic modulus, differs significantly from early differentiating mESCs regardless of cell line (R1 or D3 mESCs) or treatment (Fig. 4).

Nuclear deformability (plasticity) has been shown to decrease as stem cells differentiate (Pajerowski et al., 2007). Because the majority of an embryonic stem cell is comprised of the nucleus, alterations observed in the cells' global elastic modulus may directly reflect these changes in their nuclear fluidity. This nuclear fluidity, in turn, is affected by changes in the structural lamin components that are known to occur as ESCs differentiate (Constantinescu et al., 2006). Lamins act as dynamic molecular scaffolds within the nucleus. This 
Table 1. Average Elastic Modulus of Undifferentiated and Differentiating ESCs

\begin{tabular}{|c|c|c|c|}
\hline Line-treatment ${ }^{\mathrm{a}}$ & $\begin{array}{c}\text { Undifferentiated } \\
M e a n \mathrm{kPa} \pm S E(\mathrm{n})\end{array}$ & $\begin{array}{c}\text { Differentiating } \\
M e a n k P a \pm S E(\mathrm{n})\end{array}$ & Fold change \\
\hline R1-LIF removal (live) & $0.265 \pm 0.015(20)$ & $0.579 \pm 0.400(20)^{\mathrm{c}}$ & 2.2 \\
\hline $\begin{array}{l}\text { R1-LIF removal } \\
\text { (Fixed and assessed } \\
\text { within } 72 \mathrm{~h} \text { ) }\end{array}$ & $19.18 \pm 0.95(20)$ & $78.05 \pm 4.05(20)^{\mathrm{d}}$ & 4.0 \\
\hline $\begin{array}{l}\text { R1-LIF removal } \\
\text { (Fixed and assessed } \\
\text { within } 24 \mathrm{~h} \text { ) }\end{array}$ & $3.72 \pm 0.16(78)$ & $11.19 \pm 0.89(78)^{\mathrm{e}}$ & 3.0 \\
\hline $\begin{array}{l}\text { D3-LIF removal } \\
\text { (Fixed and assessed } \\
\text { within } 24 \mathrm{~h} \text { ) }\end{array}$ & $7.86 \pm 0.52$ & $15.66 \pm 0.63(20)^{\mathrm{e}}$ & 2.0 \\
\hline $\begin{array}{l}\text { R1-Ascorbic acid } \\
\text { (Fixed and assessed } \\
\text { within } 24 \mathrm{~h} \text { ) }\end{array}$ & $3.80 \pm 0.25(20)$ & $10.50 \pm 0.38(20)^{\mathrm{e}}$ & 2.8 \\
\hline
\end{tabular}

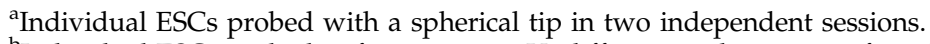

${ }^{\mathrm{b}}$ Individual ESCs probed in four sessions. Undifferentiated were significantly more supple than differentiating ESCs (Kruskal-Wallis test: ${ }^{c} p=0.003 ;{ }^{d} p=0.0002$; Mann-Whitney test: $\left.{ }^{\mathrm{e}} p<0.0001\right)$.

scaffold interacts with other proteins that are involved in chromatin interactions affecting DNA replication, chromatin modifications, and gene expression (Goldmann, 2002; Shumaker et al., 2003). Consequently, the composition of the scaffold may vary among cell types depending on the expression of various lamin isoforms and may contribute to the determination of the specific functions of a cell (Dechat et al., 2008. 2009). This suggestion is supported by our findings that both the elastic modulus and lamin A/C protein ex-

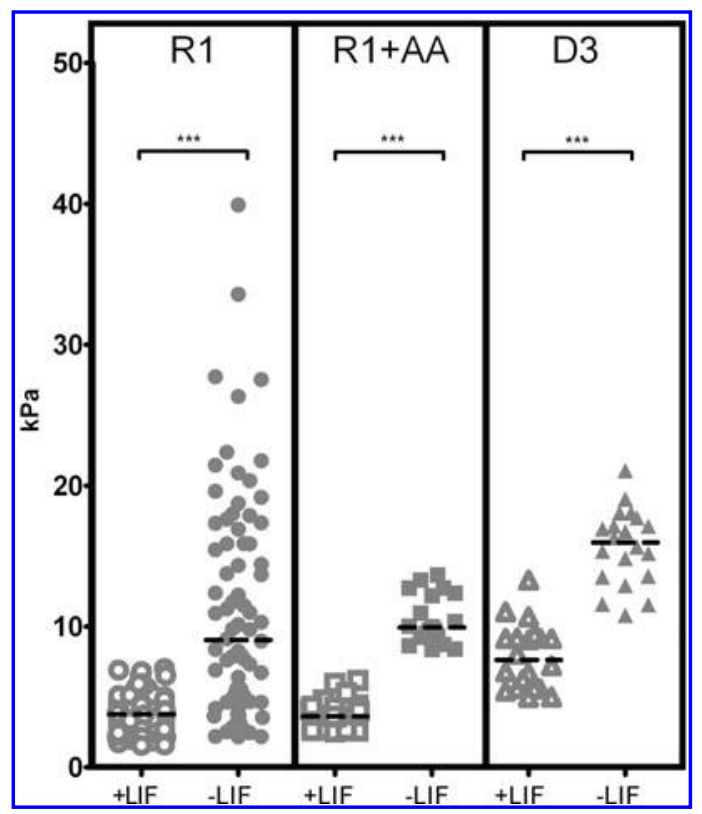

FIG. 4. Elastic modulus $(\mathrm{kPa})$ of individually measured undifferentiated (open symbols) and differentiating (filled symbols) cells following LIF removal for R1 and D3 mESC lines and LIF removal plus ascorbic acid for R1 cell line, R1 + AA. Cells assessed within $24 \mathrm{~h}$ of formaldehyde fixation. In each case, the differentiating ESCs had a significantly higher relative $\mathrm{kPa}$ than undifferentiated $(* * * p<0.0001)$. pression increased with mESC differentiation. An increase in expression of lamin $\mathrm{A} / \mathrm{C}$ is consistent with other studies that indicate that lamin $\mathrm{A} / \mathrm{C}$ expression is correlated with cell differentiation (Constantinescu et al., 2006; Pajerowski et al., 2007; Rober et al., 1989; Stewart and Burke, 1987). Changes in the cytoskeleton component, F-actin, have also been reported during ESC differentiation (Chowdhury et al., 2010). In our studies, some differentiating cells displayed a more defined pattern of F-actin stress fibers (Fig. 2), similar to cytoskeletal changes observed by others (Chowdhury et al., 2010; Hammerick et al., 2011). Therefore, alterations in both nuclear and cytoskeletal composition may contribute to the overall mechanical properties of cells and reflect changes that occur during cell differentiation. In an intriguing study, particle tracking microrheology of human induced pluripotent stem cells (iPSCs) and hECSs revealed that there were also differences in the viscoelastic properties of the cytoplasm of human fibroblasts, iPSCs, and hESCs (Daniels et al., 2010). The cytoplasm of iPSC was found to be purely viscous, whereas the cytoplasm of hESCs was predominantly viscous with some elastic subcellular regions, and that of adult fibroblasts was predominately elastic (Daniels et al., 2010). Whether the differences between hESCs and iPSCs were due to reprogramming of iPSCs to a more pluripotent ground state by the continued forced expression of the transduced pluripotency factors or were the result of inappropriate reprogramming remains to be determined.

In summary, changes in cell stiffness could be detected using AFM prior to obvious changes in cell morphology or visual subjective changes in Nanog protein level as detected by ICC, although a decrease in the percentage of cells expressing a Nanog promoter-driven reporter gene within the same 6-day time period could be observed. However, monitoring of the reporter gene was confounded by ongoing transgene silencing. Measurements of the elastic modulus should allow us to define early events in ESC differentiation using a minimally invasive, single-touch technique and without the problems associated with reporter trangenes (Stewart et al., 2008). Although real-time monitoring of cell elasticity can be used to detect dynamic cellular responses to 
changes in chemical or mechanical environment (Schillers et al., 2010), cyclic applications of force to mechanically sensitive stem cells have been shown to trigger cell differentiation (Chowdhury et al., 2010). Single indentation probing using AFM involves less cell manipulation than other methods used to characterize cell mechanical properties, such as the rheological aspiration or twisted bead approach, and as such, may be less likely to trigger unwanted cell responses. Improvements in AFM efficiencies will allow processing of large numbers of cells within shorter time frames, thus making AFM an attractive and experimentally tractable method by which to analyze ESC differentiation.

Our results demonstrate that mechanical phenotyping through AFM has the potential to be either scaled-up for translational purposes or, contrarily, used to focus on single live cells and follow changes in mechanical properties overtime under specific induction/dedifferentiation conditions. Mechanical phenotyping should prove to be a valuable tool in the development of improved methods of targeted cellular differentiation and/or reprogramming of human embryonic, iPS and adult stem cells for therapeutic purposes, for development of new diagnostic procedures, and to monitor cellular responses to environmental stimuli.

\section{Acknowledgments}

We thank Lei Li and Rajarshi Roy for help with cell culture and AFM probing. This work was supported by National Science Foundation grants CMMI 0826158 and IIS 0711038.

\section{Author Disclosure Statement}

The authors declare that no conflicting financial interests exist.

\section{References}

Alcaraz, J., Buscemi, L., Grabulosa, M., et al. (2003). Microrheology of human lung epithelial cells measured by atomic force microscopy. Biophys. J. 84, 2071-2079.

Bilodeau, G.G. (1992). Regular pyramid punch problem. I. Appl. Mech. 59, 519-523.

Bourne, S., Polak, J.M., Hughes, S.P.F., et al. (2004). Osteogenic differentiation of mouse embryonic stem cells: differential gene expression analysis by cDNA microarray and purification of osteoblasts by cadherin-11 magnetically activated cell sorting. Tissue Eng. 10, 796-806.

Cao, Y., Yang, D., and Soboyejoy, W. (2005). Nanoindentation method for determining the initial contact and adhesion characteristics of soft polydimethylsiloxane. J. Mater. Res. 20, 2004-2011.

Chambers, I., and Smith, A. (2004). Self-renewal of teratocarcinoma and embryonic stem cells. Oncogene 23, 7150-7160.

Chowdhury, F., Na, S., Li, D., et al. (2010). Material properties of the cell dictate stress-induced spreading and differentiation in embryonic stem cells. Nat. Mater. 9, 82-88.

Christoforou, N., Miller, R.A., Hill, C.M., et al. (2008). Mouse ES cell-derived cardiac precursor cells are multipotent and facilitate identification of novel cardiac genes. J. Clin. Invest. 118, 894-903.

Collinsworth, A.M., Zhang, S., Kraus, W.E., et al. (2002). Apparent elastic modulus and hysteresis of skeletal muscle cells throughout differentiation. Am. J. Physiol. Cell Physiol. 283, 1219-1227.
Constantinescu, D., Gray, H.L., Sammak, P.J., et al. (2006). Lamin A/C expression is a marker of mouse and human embryonic stem cell differentiation. Stem Cells 24, 177-185.

Daniels, B.R., Hale, C.M., Khatau, S.B., et al. (2010). Differences in the microrheology of human embryonic stem cells and human induced pluripotent stem cells. Biophys. J. 99, 35633570 .

Danti, S., D'Acunto, M., Trombi, L., et al. (2007). A micro/ nanoscale surface mechanical study on morpho-functional changes in multilineage-differentiated human mesenchymal stem cells. Macromol. Biosci. 7, 589-598.

Dechat, T., Pfleghaar, K., Sengupta, K., et al. (2008). Nuclear lamins: major factors in the structural organization and function of the nucleus and chromatin. Genes Dev. 22, 832-853.

Dechat, T., Adam, S.A., and Goldman, R.D. (2009). Nuclear lamins and chromatin: when structure meets function. Adv. Enzyme Regul. 49, 157-166.

Docheva, D., Padula, D., Popov, C., et al. (2008). Researching into the cellular shape, volume and elasticity of mesenchymal stem cells, osteoblasts and osteosarcoma cells by atomic force microscopy. J. Cell Mol. Med. 12, 537-552.

Donovan, P.J., and Gearhart, J. (2001). The end of the beginning for pluripotent stem cells. Nature 414, 92-97.

Girot, M., Boukallel, M., and Régnier, S. (2006). Modeling soft contact mechanism of biological cells using an atomic force bio-microscope. In International Conference on Intelligent Robots and Systems, Beijing, China, pp. 1831-1836.

Goldmann, W.H. (2002). Mechanical aspects of cell shape regulation and signaling. Cell Biol. Int. 26, 313-317.

Hammerick, K.E., Huang, Z., Sun, N., et al. (2011). Elastic properties of induced pluripotent stem cells. Tissue Eng Part A 17, 495-502.

He, S., Pant, D., Schiffmacher, A., et al. (2006). Developmental expression of pluripotency determining factors in caprine embryos: novel pattern of NANOG protein localization in the nucleolus. Mol. Reprod. Dev. 73, 1512-1522.

He, S., Pant, D., Schiffmacher, A., et al. (2008). Lymphoid enhancer factor 1-mediated Wnt signaling promotes the initiation of trophoblast lineage differentiation in mouse embryonic stem cells. Stem Cells 26, 842-849.

Hutter, J.L., and Bechhoefer, J. (1993). Calibration of atomic force microscope tips. Rev. Sci. Instrum. 64, 1868-1873.

Johnson, K.L. (1982). One hundred years of Hertz contact. In Proc Inst Mech Eng 196.

Kolossov, E., Fleischmann, B.K., Liu, Q., et al. (1998). Functional characteristics of ES cell-derived cardiac precursor cells identified by tissue specific expression of the green fluorescent protein. J. Cell Biol. 143, 2045-2056.

Kwon, E., Kim, Y., Park, J., et al. (2007). Measurement of pull-off forces by atomic force microscope in liquids used for biological applications. Exp. Tech. 31, 40-45.

Ladjal, H., Hanus, J.L., Pillarisetti, A., et al. (2010). Reality-based real-time cell indentation stimulator. IEEE/ASME Trans. Mech. PP, 1-12.

Maugis, D. (2000). Contact, Adhesion and Rupture of Elastic Solids (Springer, New York).

Nagano, K., Yoshida, Y., and Isobe, T. (2008). Cell surface biomarkers of embryonic stem cells. Proteomics 8, 4025-4035.

Odorico, J.S., Kaufman, D.S., and Thomson, J.A. (2001). Multilineage differentiation from human embryonic stem cell lines. Stem Cells 19, 193-204.

Pajerowski, J.D., Dahl, K.N., Zhong, F.L., et al. (2007). Physical plasticity of the nucleus in stem cell differentiation. Proc. Natl. Acad. Sci. USA 104, 15619-15624. 
Pillarisetti, A. (2009). Mechanical Manipulation and Characterization of Biological Cells (University of Maryland, College Park, MD).

Pillarisetti, A., Ladjal, H., Ferreira, A., Keefer, C., and Desai, J.P. (2009). Mechanical characterization of mouse embryonic stem cells. In 31st Annual International Conference of the IEEE Engineering in Medicine and Biology Society, Minneapolis, Minnesota, pp. 1176-1179.

Pillarisetti, A., Keefer, C., and Desai, J.P. (2008). Mechanical response of embryonic stem cells using haptics-enabled atomic force microscopy. In International Symposium on Experimental Robotics, Athens, Greece.

Rico, F., Roca-Cusachs, P., Gavara, N., et al. (2005). Probing mechanical properties of living cells by atomic force microscopy with blunted pyramidal cantilever tips. Phys. Rev. E 72, $1-10$.

Rober, R.A., Weber, K., and Osborn, M. (1989). Differential timing of nuclear lamin $\mathrm{A} / \mathrm{C}$ expression in the various organs of the mouse embryo and the young animal: a developmental study. Development 105, 365-378.

Rosenbluth, M.J., Lam, W.A., and Fletcher, D.A. (2006). Force microscopy of nonadherent cells: a comparison of leukemia cell deformability. Biophys. J. 90, 2994-3003.

Schillers, H., Walte, M., Urbanova, K., et al. (2010). Real-time monitoring of cell elasticity reveals oscillating myosin activity. Biophys. J. 99, 3639-3646.

Sharova, L.V., Sharov, A.A., Piao, Y., et al. (2007). Global gene expression profiling reveals similarities and differences among mouse pluripotent stem cells of different origins and strains. Dev. Biol. 307, 446-459.

Shumaker, D.K., Kuczmarski, E.R., and Goldman, R.D. (2003). The nucleoskeleton: lamins and actin are major players in essential nuclear functions. Curr. Opin. Cell Biol. 15, 358-366.

Smith, A.G. (2001). Embryo-derived stem cells: of mice and men. Annu. Rev. Cell Dev. Biol. 17, 435-462.
Sneddon, I.N. (1948). Boussinesq's problem for a rigid cone. Math. Proc. Cambridge Philos. Soc. 44, 492-507.

Stewart, C., and Burke, B. (1987). Teratocarcinoma stem cells and early mouse embryos contain only a single major lamin polypeptide closely resembling lamin B. Cell 51, 383-392.

Stewart, R., Yang, C., Anyfantis, G., e al. (2008). Silencing of the expression of pluripotent driven-reporter genes stably transfected into human pluripotent cells. Regen. Med. 3, 505-522.

Takahashi, T., Lord, B., Schulze, P.S., et al. (2003). Ascorbic acid enhances differentiation of embryonic stem cells into cardiac myocytes. Basic Sci. Rep. 107, 1912-1916.

Titushkin, I., and Cho, M. (2007). Modulation of cellular mechanics during osteogenic differentiation of human mesenchymal stem cells. Biophys. J. 93, 3693-3702.

Touhami, A., Nysten, B., and Dufrêne, Y.F. (2003). Nanoscale mapping of the elasticity of microbial cells by atomic force microscopy. Langmuir 19, 4539-4543.

Vinckier, A., and Semenza, G. (1998). Measuring elasticity of biological materials by atomic force microscopy. FEBS Lett. 430, 12-16.

Weisenhorn, A.L., Khorsandi, M., Kasas, S., et al. (1993). Deformation and height anomaly of soft surfaces studied with an AFM. Nanotechnology 4, 106-113.

Yourek, G., Hussain, M.A., and Mao, J.J. (2007). Cytoskeletal changes of mesenchymal stem cells during differentiation. Asaio J. 53, 219-228.

Address correspondence to: Carol L. Keefer Department of Animal and Avian Sciences University of Maryland Bldg 142, rm 2129 College Park, MD 20742

E-mail: ckeefer@umd.edu 